Proceedings of the 1998 IEEE

International Conference on Robotics \& Automation

Leuven, Belgium • May 1998

\title{
ROBOTIC MANIPULATION OF OPHTHALMIC LENSES ASSISTED BY A DEDICATED VISION SYSTEM.
}

\author{
X. Fernàndez and J. Amat** \\ ”Engineering Dept., Industrias de Óptica (INDO), L'Hospitalet (Barcelona), Spain. E-mail: xavier@indo.es. \\ ** Automatic Control Dept., Universitat Politècnica de Catalunya (UPC), Barcelona, Spain. E-mail: amat@esaii.upc.es.
}

\begin{abstract}
A robotic system developed to manipulate ophthalmic lenses during the edging process is presented. The robot is assisted by a machine vision system to accurately determine the position and orientation of the lens optical center and axis, through the localization of two small fiducial marks on the lens surface. The optimal design of the fiducial marks is investigated in order to minimize their size. It is shown that a regular diamond is the most detectable shape at minimum sizes. Three different camera placement strategies are discussed, taking into account accuracy constraints and calibration requirements; the chosen solution uses a camera attached to the robot end-effector. The robotic system proves to be advantageous with respect to the manual process, substantially improving the final accuracy while maintaining or even reducing the cycle time.
\end{abstract}

\section{INTRODUCTION}

One of the final steps in ophthalmic lens production is the edging of the disk-shaped manufactured lens in order to fit the particular shape of the spectacle's frame. This edging process is performed by a special milling machine.

The key factor for the success of this process is the accurate positioning of the prescribed optical center and optical axis of the lens with respect to the frame's shape. Thus, the placement of the lens on the blocking grip of the edging machine has a tight requirement of positional and angular precision.

The loading of the lens on the edging machine is usually a manual operation, where the lens alignment is visually estimated. This kind of manual procedure is subject to a high level of variability and subjectivity, resulting in a general lack of accuracy, and showing frequent out-oftolerance positioning errors.
In order to solve this problem, we present in this paper a robotic system which manipulates the lenses to load and discharge the edging machine. To robotize the process and to ensure accuracy, a machine vision system have been added to assist the robot manipulator. This vision system bases its operation on the localization of two small fiducial marks placed on the lens surface. The shape and size of these marks has been studied in order to be used with proficiency by the vision system while reducing their visibility for the lens user.

\section{SYSTEM STRUCTURE}

\subsection{Layout}

The disk-shaped lenses, outgoing from the previous polishing step, are fed to the automated working station by means of an input conveyor belt $\mathbf{T}_{\mathbf{1}}$ (fig. 1). The robot $\mathbf{R}$ executes the loading and discharge of the edging machine EM. Processed lenses are finally sent to the subsequent station (for packing and storing) by the output
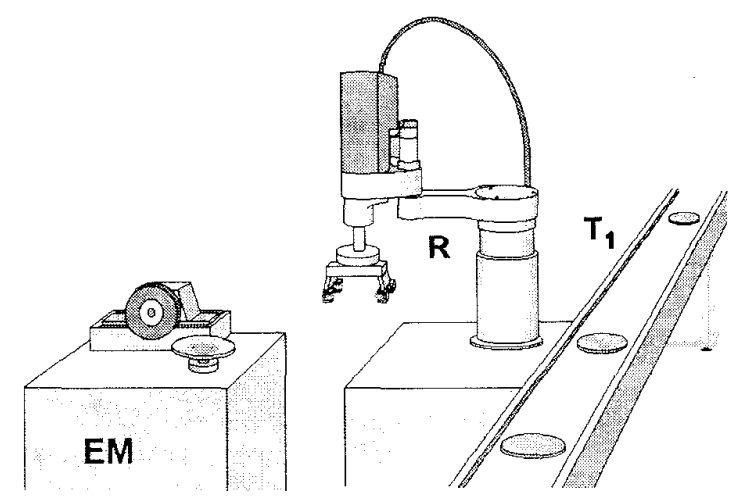

Fig. 1: System layout. 
conveyor belt $\mathbf{T}_{2}$.

The time of operation of the station is mainly determined by the edging time of the lens in the edging machine EM, which is comprised between 30 and 50 seconds, depending on lens thickness and the particular goal shape.

In order to ensure the lens quality, it is very important to preserve its optical center and optical axis relative to the frame shape. Thus, the operation of loading the lens to the EM machine, performed by the robot, requires a high degree of accuracy in position and orientation.

\subsection{The edging machine.}

The edging machine $\mathbf{E M}$ is used to cut the edge of the lenses to obtain the outline of the spectacle's frame (fig.2). This machine is constituted by a lens-holding spindle having two degrees of freedom, rotation $\theta$ and lifting up $\mathbf{Z}$, and a diamond wheel with one degree of freedom, advance $Y$ (fig.3).

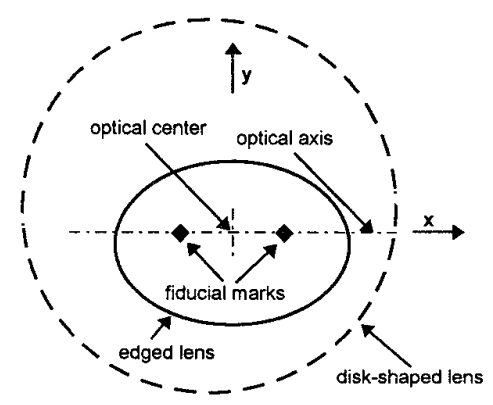

Fig. 2: Edging a round lens to the shape of the frame.

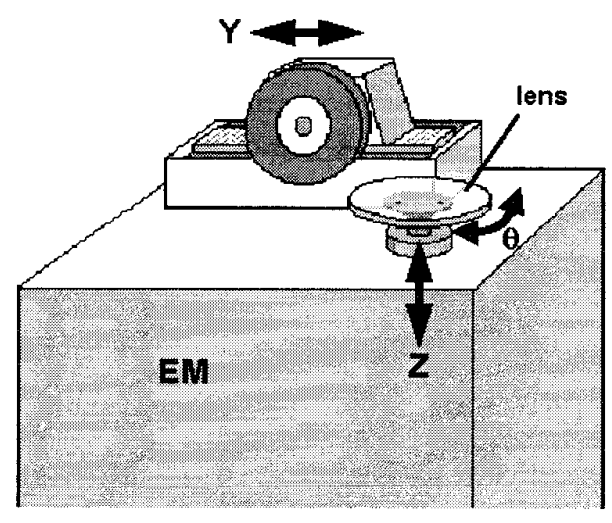

Fig. 3: The lens-edging milling machine.
The rotating speed of the wheel is about $6000 \mathrm{rpm}$, and the working speed is determined according to the lens thickness. Lens-holding spindle rotation can be programmed to advance step by step or continuously at a consigned speed. Usual working speed ranges from 2 to 5 $\mathrm{cm} / \mathrm{sec}$, which yields the above mentioned edging time of 30 to $50 \mathrm{sec}$. per lens.

The alignment accuracy of the lens on the holding spindle must be better than $0.5 \mathrm{~mm}$ in position and $0.3 \mathrm{deg}$. in orientation, in order to maintain the optical properties of the edged lens.

\subsection{The vision system.}

When the lenses enter the working station on the conveyor belt $\mathbf{T}_{1}$, they show an arbitrary position and an approximate but not precise orientation. It is necessary, however, that the robot place them at a fixed and accurate position and orientation on the EM lens-holding spindle, referred to the lens optical center and axis. Thus, we have decided to use a machine vision system in order to detect the arrival of each lens and to obtain a precise measure of its position and orientation through the determination of the lens optical center and axis.

Lens optical center and axis are defined from the optical properties of the upper and lower surfaces of the lens, and it is not possible to relate them to visible geometrical features on the disk-shaped lens, because it is transparent and its contour is inaccurate, and optical center does not correspond to the geometric center of the disk.

To solve this problem, in the early steps of manufacturing two small fiducial marks are printed or engraved on the upper surface, in order to determine the optical center and axis from the position of these fiducial marks (fig.2). The size of the marks is a critical limitation: it must be kept as small as possible in order not to interfere with the field of view of the optical instruments which are used during production.

The vision system, consequently, must be able to locate these small fiducial marks with reliability and accuracy. Processing time, however, is not critical because image acquisition and processing is performed in parallel to the edging of the previous lens.

\section{DETECTABILITY AND ACCURACY ACHIEVED BY THE VISION SYSTEM}

\subsection{Vision system configurations.}

There are three possible strategies for the proposed vision system (fig.4): 


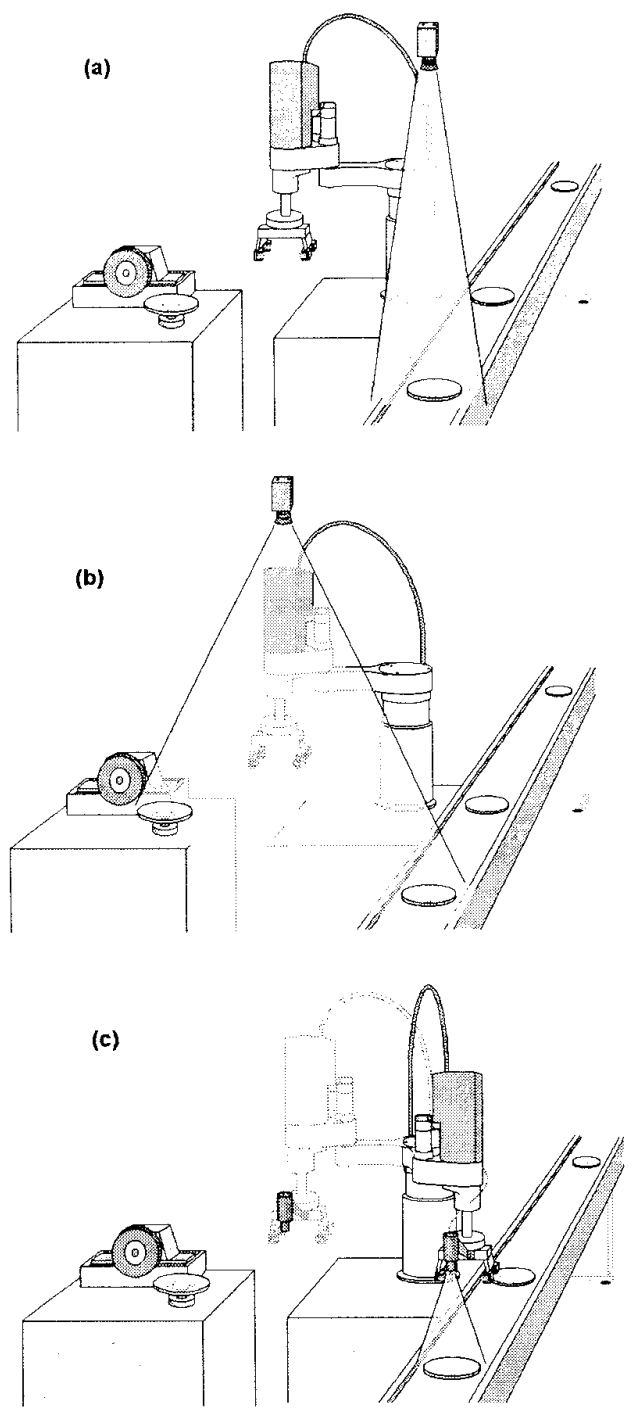

Fig. 4: Three possible vision system configurations.

a) The camera may be located over the input conveyor belt $\mathbf{T}_{1}$ in order to obtain the position and orientation of each lens. This solution requires a periodic calibration process in order to compensate for casual relative displacements of the camera with respect to the EM machine and the robot $\mathbf{R}$.

b) The camera can be fixed at a point above the working station, in such a way that a complete scene is acquired, including the input lenses on the conveyor $T_{1}$, and the EM holding spindle where the lens has to be loaded. In this case, the movement to be executed by the robot would be a function of the relative displacement between two positions obtained from a single scene [2]. A calibration is required again to relate the position of the camera to the axis of the robot.

c) The camera can be attached to the robot's end effector, [3]. With this placement, the input lens image is acquired in similar conditions as strategy $\boldsymbol{a}$, but an additional image of the EM lens-holding spindle can be acquired periodically to aid the calibration process.

Strategy $a$ requires a complex calibration process between the camera, the robot $\mathbf{R}$ and the edging machine. Strategy $b$ needs a more simple calibration between the camera and the robot, but it has the disadvantage of obtaining more reduced images, thus worsening the measurement accuracy.

On the other hand, strategy $c$ requires the calibration of the robot with respect to the EM machine, but this operation can be executed periodically, through the visualization of two reference marks placed on the EM lens-holding spindle. This reason has led strategy $c$ to be chosen, despite the difficulties that arise when a camera has to be attached to a robot.

\subsection{Design of the fiducial marks.}

In order to design the proper shape for the fiducial marks placed on the lens, we have to take into account the goal of minimizing their size while obtaining a high detection reliability and a good positional precision for the resulting optical center and axis.

A major constraint have to be regarded: the detection of the marks is conditioned by a complex scene, caused by the reflections on the lens surface of the surrounding objects and lighting.

The usual method for detecting small objects of known shape in a scene is template matching [4],[5]. Every portion of the image is compared with a model of the object previously acquired from a prototype scene. Detection of the object is assumed at the places where the local similarity between the model and the image exceeds a threshold. Similarity is computed by means of a similarity measure, and normalized correlation is one of the most extended and useful.

Detection of small marks in a complex scene is conditioned by two opposite factors: the loss of similarity between model and mark because the poor resolution (which can prevent detection), and the partial similarity between the model and the background objects (cause of false detection) [6]. This is a discrimination problem 
which bounds the minimum useful size for a fiducial mark.

The relationship between mark shape, minimum size and discrimination has been investigated [7], [8]. We have considered the following shapes:

\section{$0 \bigcirc+\square \oplus \square \bullet$}

In order to compare discrimination capability, we look for the minimum mark size for which the similarity between the model and a mark at an adverse position (solid line, figs. 5 and 6) exceeds the partial similarity with respect to a simulated background, composed by edges and vertices (dotted line). Sizes are measured in pixel units on the image formed on the CCD sensor of the camera, and similarity values are in normalized correlation units. A regular diamond discriminates the best among the other shapes, as can be seen in fig. 5, where the minimum theoretical discriminable size is $4 \times 4$ pixels. Next shape in order of discrimination capability is a cross with $5 \times 5$ pixels, and a square (fig. 6) yields the worst case, $8 \times 8$ pixels.

In a practical situation it is wise to keep some

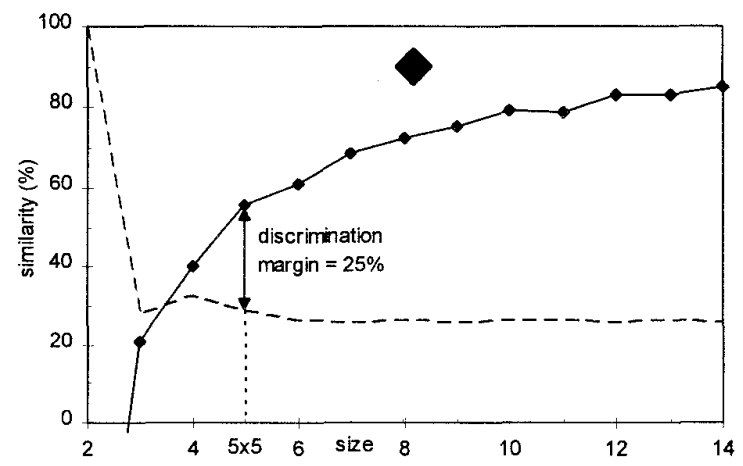

Fig. 5: Detection and discrimination plot for diamond.

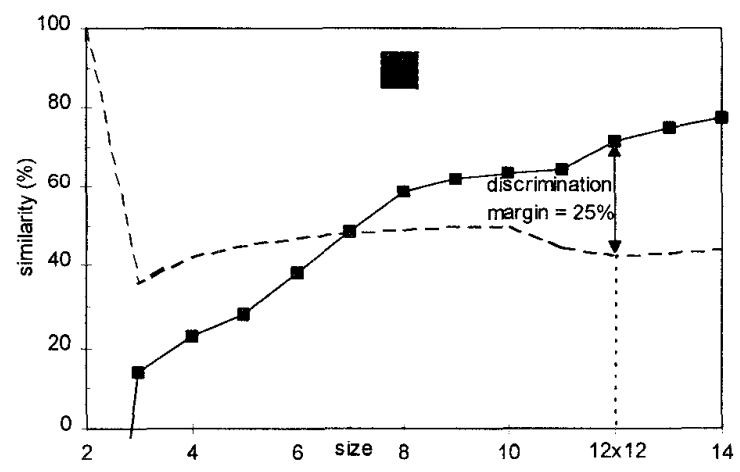

Fig. 6: Detection and discrimination plot for square. discrimination margin (vertical distance between both curves in figs. 5 and 6), to cope with small shape defects and noise. Table 1 shows the minimum discriminable size for the seven shapes, for a margin of $25 \%$. From this table, we propose to use diamond-shaped, $5 \times 5$ pixels size fiducial marks in order to achieve the goal of minimum size.

\begin{tabular}{|c|c|}
\hline SHAPE & MINIMUM SIZE \\
\hline & $5 \times 5$ pixels \\
\hline & $7 \times 7$ pixels \\
\hline & $7 \times 7$ pixels \\
\hline & $9 \times 9$ pixels \\
\hline 0 & $9 \times 9$ pixels \\
\hline$\square$ & $11 \times 11$ pixels \\
\hline & $12 \times 12$ pixels \\
\hline
\end{tabular}

Table 1: Minimum discriminable sizes for a $25 \%$ margin.

\subsection{Positional accuracy.}

Template matching techniques locate the position of the marks within 1 pixel accuracy. When a higher precision is required, the method of centroid can be used. Bose and Amir [9], show that for small-size fiducial marks (less than $10 \times 10$ pixels) this method yields a maximum error of 0.3 pixels (in any direction), independently of the shape. Thus, this is the positional accuracy we can expect for the localization of the fiducial marks.

Using $c$ strategy, a $150 \mathrm{~mm}$-wide scene (to include the whole lens disk and possible displacements) is imaged onto a 400 pixel-wide CCD sensor; thus the 0.3 pixel accuracy corresponds to a maximum error of $0.12 \mathrm{~mm}$ for the optical center. On the other hand, the prescribed distance between marks is $50 \mathrm{~mm}$; this results in a 0.15 deg. error for the optical axis of the lens.

In this application, a Yamaha robot YK 640 is used, with SCARA architecture. Its accuracy is $0.2 \mathrm{~mm}$ in position and $0.1 \mathrm{deg}$. in orientation. The main error source, however, emanates from the small lens displacements when it is grasped and released.

In order to reduce the grasping error, the end effector has been built on the basis of a four-finger, parallel sliding grip (fig 7), which self-centers the lens when it is grasped. 


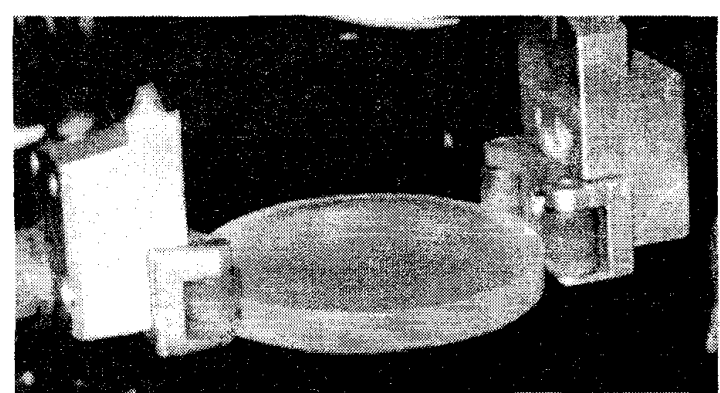

Fig. 7: The four-finger grip.

The releasing error has been minimized by means of the application of vacuum to the lens holder of the EM spindle before the lens release from the robot grip.

With these strategies, the manipulation errors have been kept at values similar to those that can be expected for the robot. Combining all sources of error, visions system localization and robot positioning and manipulation, the prescribed maximum tolerable errors for optical center and axis (fig. 8) will not be exceeded.

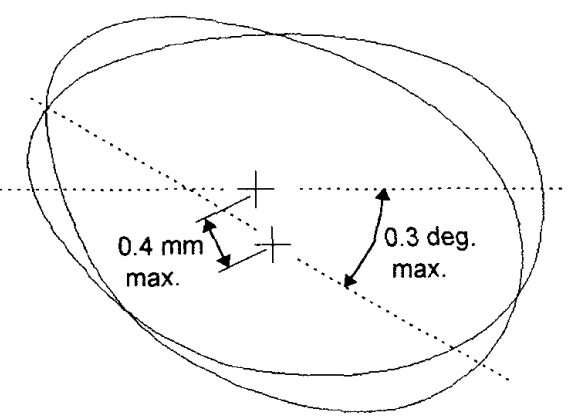

Fig. 8: Maximum tolerable position and orientation errors for optical center and optical axis.

\section{EVALUATION OF THE SYSTEM}

The performance of the robotic system has been compared to the manual operation, taking into account two factors: time of operation and accuracy.

Fig. 9 displays the operation time required for a typical lens, comparing manual and robotized processes. It can be seen that edging time is the same for both systems, while there is a small time saving in the robotized loading and unloading operations.

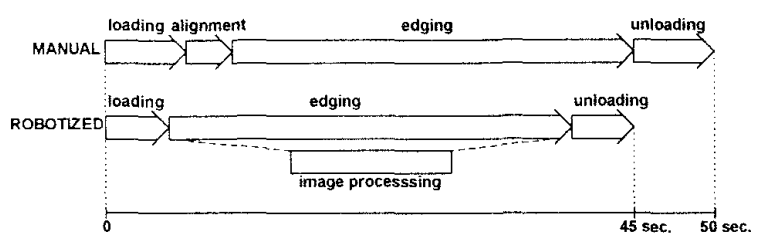

Fig. 9 : Comparison of operating time between the manual system and the robotic system.

The main time reduction comes from the absence of a visual alignment step, which is required in the manual case. Instead, the robot can acquire and process the image of the next lens during the machining of the previous one. The total time reduction amounts to $5 \mathrm{sec}$. in a cycle of 50 sec. (a 10\% saving).

Concerning to the accuracy of the resulting optical center and axis, the position and orientation error have been measured on the edged lenses, in two series of samples corresponding to the manual and the robotic system (fig. 10). It can be observed that, in the manual case, the position error is usually less than $0.4 \mathrm{~mm}$, but there are frequent outliers up to $0.9 \mathrm{~mm}$ of error. In the robotized case, the error is always bounded to $0.3 \mathrm{~mm}$. A similar behavior is observed for the orientation error (fig. 10). In
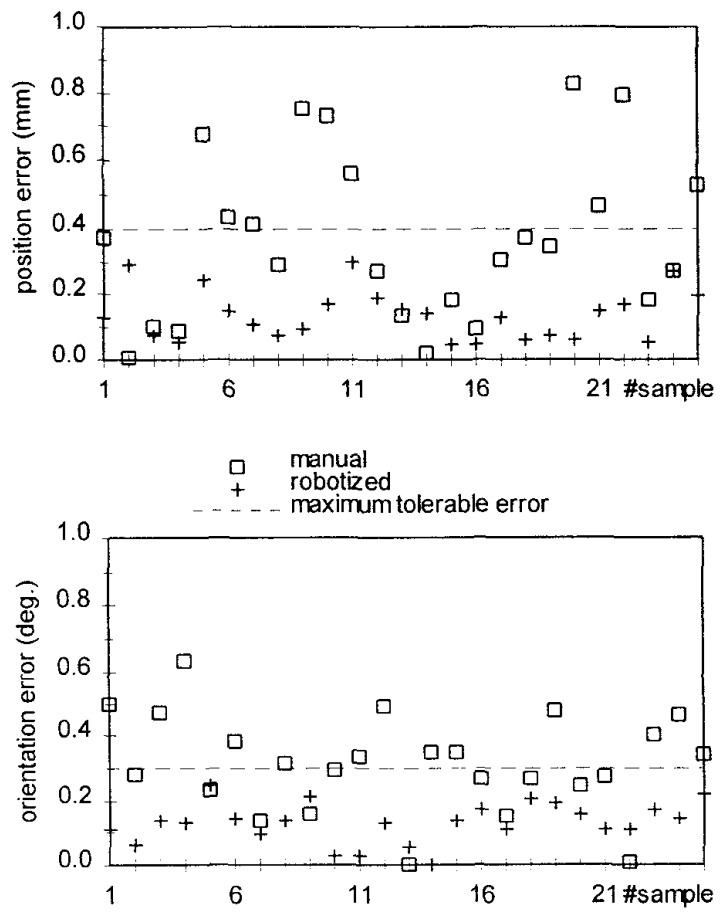

Fig. 10: Position and orientation errors for the manual system and the robotic system. 
the robotized system, both errors fulfill the goal specification for accuracy.

\section{CONCLUSIONS}

The strategy of attaching the camera to the robot end effector has proven to be an advantageous solution, because it yields a high image resolution to locate the fiducial marks on the lens, while it allows an automatic referencing of the coordinate system, and at the same time avoids a complex periodic calibration process.

The mean position and orientation error values of the edged-lens optical center and axis have been substantially reduced in the robotized system with respect to the manual operation. A major fraction of this accuracy improvement is due to the absence of outlier errors, which are common to the manual alignment procedures.

This accuracy improvement has been achieved with no increase of the operation time; in fact, this time has been reduced because the absence of the manual alignment step. A small decrease in transportation operation time has been also achieved.

\section{References:}

[1] A. Nikolaev and S.K. Nayar. "Transparent Grippers for Robot Vision". IEEE Int. Conf. on Robotics and Automation. Mineapolis, 1996.

[2] G.S. Bell and W.J. Wilson "Coordinate Controller Design for Position Based Visual Servoing in Cartesian Coordinates". IEEE Int. Conf. on Robotics and Automation. Mineapolis, 1996.
[3] N.P. Papanikolopoulos, P.K. Kosla and T. Kanade "Visual tracking of a moving target by camera mounted of a robot: A combination of control and vision". IEEE Transactions. on Robotics and Automation, Vol. 9 Feb. 1993.

[4] B.K.P. Horn "Robot Vision" Mc. Ggaw-Hill, New York 1986

[5] A. Hill, C.J. TaylorT.F. Cootes "Object Recognition by Flexible Template Matching using Genetic Algorithms". Proc. European Conf. on Computer Vision, ECCV'92

[6] W.V.K. Vijaya Kumar, D. W. Carlson and A. Mahalanobis. "Optimal trade-off syntetic discriminant funtion filters for arbitrary devices". Optical Letters 16, 1991

[7] X. Fernandez, "Template matching of binary tatgets in gray-scale images: a nonparametric approach", Pattern Recognition, Vol 30, n7, pp.1175-1182 (1997).

[8] X. Fernandez, J. Amat, "Detection of small-size synthetic references acquired with a pixelated device", Optical Engineering 36 (11), pp. 30733080 (1997).

[9] C.B. Bose, I. Amir. "Design of Fiducials for Accurate Registration Using Machine Vision", IEEE Trans. PAMI, Vol. 12, n 12, pp. 1196-1200, 1990.

[10] S. Okada, M Imade, H. Miyauchi T. Sumimoto and H. Yamamoto. "Automatic Identification of Conveyer -Transferred Parts through Image Data Prosessing". 20th Int. Conf. on Industrial Electronics Control and Instrumentation, IECON'94, Bologna, Italy, 1994. 